\title{
Visualization of relativistic laser pulses in underdense plasma
}

\author{
M. B. Schwab $\odot,{ }^{1,2, *}$ E. Siminos ${ }^{3}$ T. Heinemann $\odot,{ }^{4,5,6}$ D. Ullmann $\odot,{ }^{4,5}$ F. Karbstein, ${ }^{2,7}$ \\ S. Kuschel, ${ }^{1,2,8}$ A. Sävert, ${ }^{1,2}$ M. Yeung $\oplus^{2,9}$ D. Hollatz, ${ }^{1,2}$ A. Seidel® ${ }^{1,2}$ J. Cole, ${ }^{10}$ \\ S. P. D. Mangles ${ }^{10}{ }^{10}$ B. Hidding, ${ }^{4,5}$ M. Zepf, ${ }^{1,2,9}$ S. Skupin, ${ }^{11}$ and M. C. Kaluza ${ }^{1,2}$ \\ ${ }^{1}$ Institute of Optics and Quantum Electronics, Friedrich-Schiller-Universität Jena, Jena 07743, Germany \\ ${ }^{2}$ Helmholtz-Institute Jena, Jena 07743, Germany \\ ${ }^{3}$ University of Gothenburg, Department of Physics, SE412 96 Gothenburg, Sweden \\ ${ }^{4}$ Scottish Universities Physics Alliance, Department of Physics, University of Strathclyde, \\ Glasgow G4 ONG, United Kingdom \\ ${ }^{5}$ Cockcroft Institute, Sci-Tech Daresbury, Keckwick Lane, Daresbury, \\ Cheshire WA4 4AD, United Kingdom \\ ${ }^{6}$ Deutsches Elektronen-Synchrotron DESY, Hamburg 22607, Germany \\ ${ }^{7}$ Theoretisch-Physikalisches Institut, Friedrich-Schiller-Universität, Jena 07743, Germany \\ ${ }^{8}$ SLAC National Accelerator Lab, 2575 Sand Hill Road, Menlo Park, California 94025, USA \\ ${ }^{9}$ School of Mathematics and Physics, Queens University Belfast, BT7 1NN, United Kingdom \\ ${ }^{10}$ The John Adams Institute for Accelerator Science, Blackett Laboratory, Imperial College London, \\ London SW7 2AZ, United Kingdom \\ ${ }^{11}$ Institut Lumière Matière, CNRS, Université de Lyon, Lyon 69352, France
}

(Received 10 September 2019; revised manuscript received 22 November 2019; accepted 10 February 2020; published 2 March 2020)

\begin{abstract}
We present experimental evidence of relativistic electron-cyclotron resonances (RECRs) in the vicinity of the relativistically intense pump laser of a laser wakefield accelerator (LWFA). The effects of the RECRs are visualized by imaging the driven plasma wave with a few-cycle, optical probe in transverse geometry. The probe experiences strong, spectrally dependent and relativistically modified birefringence in the vicinity of the pump that arises due to the plasma electrons' relativistic motion in the pump's electromagnetic fields. The spectral birefringence is strongly dependent on the local magnetic field distribution of the pump laser. Analysis and comparison to both 2D and 3D particle-in-cell simulations confirm the origin of the RECR effect and its appearance in experimental and simulated shadowgrams of the laser-plasma interaction. The RECR effect is relevant for any relativistic, magnetized plasma and in the case of LWFA could provide a nondestructive, in situ diagnostic for tracking the evolution of the pump's intensity distribution with propagation through tenuous plasma.
\end{abstract}

DOI: 10.1103/PhysRevAccelBeams.23.032801

\section{INTRODUCTION}

For many applications based on the interaction of a high-intensity, electromagnetic (EM) pump pulse and matter, the detailed evolution of the pump is of great importance. While simulations are capable of describing this evolution in great detail, the experimental verification of these predictions must still be demonstrated when developing a robust physical understanding of the system. Lack of experimental verification is typically due to the lack of suitable diagnostics that are capable of measuring the evolution of the pump's fields or intensity in a nondestructive manner. In particular,

\footnotetext{
"mattbschwab@gmail.com
}

Published by the American Physical Society under the terms of the Creative Commons Attribution 4.0 International license. Further distribution of this work must maintain attribution to the author(s) and the published article's title, journal citation, and DOI. plasma-based particle accelerators, which have been developed over the past decades from proof-of-concept [1-3] to prototype technologies for the next generation of particle accelerators [4-7] would greatly benefit from such a diagnostic. Whether laser-driven wakefield acceleration (LWFA) or beam-driven plasma wakefield acceleration, the evolution of the pump and of the wakefield structure in the plasma is of critical importance for the resulting properties of the accelerated particles, e.g., electrons or positrons. Whereas diagnostics for characterizing the accelerated particle bunch [8-12], their associated magnetic fields [13,14], the wakefield's plasma-density distribution [15-17], and quasielectrostatic fields $[18,19]$, as well as the laser or particle driver in vacuum $[20,21]$ are available, measurement of the driver in situ has been limited to nonspatially resolved indicators in terms of the pump's local intensity distribution $[22,23]$. 
Electron-cyclotron resonances (ECRs) offer a potential means to track the strong magnetic fields of a relativistic driver in plasma. They have been used to heat fusion plasmas, e.g., electron-cyclotron resonant heating [24,25], to characterize temperature distributions of plasma electrons, e.g., electron-cyclotron emission imaging [26], and depend on the presence of underdense, magnetized plasma. ECRs also play an important role in atmospheric sciences and semiconductor manufacturing, albeit typically for static or quasistatic magnetic fields. While the physics behind electron-cyclotron motion in magnetized plasma at the millitesla to tesla level are well established, their application to laser-plasma accelerators demands essential modifications to the theory that take into account relativistic drift motion of the plasma electrons in EM fields. The focus of this paper is in describing relativistic electron-cyclotron resonances (RECRs) and how they can be exploited to realize a nondestructive imaging diagnostic that is sensitive to the evolving intensity distribution of a linearly polarized pump laser in underdense plasma.

The paper is organized as follows. Section II describes the experimental setup and Sec. III presents the experimental results. Section IV proceeds with modifying the existing Appleton-Hartree equation from magnetoionic theory to include relativistic motion of the plasma electrons which is later used to test the origin of the RECR effect. Section V presents results from a 3D particle-in-cell simulation using the EPOCH code which reproduces the RECR signals seen in the experiment. Section VI describes 2D particle-in-cell simulations using the vsim code that have the benefit of producing particle- and field-tracking data. This data is used with the relativistic Appleton-Hartree equation to further test the origin of the RECR signals seen in both the experiment and the EPOCH simulation. Finally, Sec. VII concludes the paper.

\section{EXPERIMENTAL SETUP}

Our experiments were performed at the JETI 40 Ti: sapphire laser at the Institute of Optics and Quantum Electronics in Jena, Germany. Pump pulses with a FWHM duration of $27 \mathrm{fs}$ and total energy of $650 \mathrm{~mJ}$ were focused with an $F / 12$ off-axis parabolic mirror to a $(9.7 \pm 0.1) \mu \mathrm{m}$ FWHM focus with $37 \%$ of the pulse energy inside the FWHM area on target. Assuming a Gaussian shape, the peak intensity in vacuum of $2.3 \times 10^{19} \mathrm{~W} / \mathrm{cm}^{2}$ is calculated corresponding to a peak normalized vector potential of $a_{0}=e E_{0} /\left(\omega_{L} m_{e} c\right)=3.3$, with elementary charge $e$, laser's electric field amplitude $E_{0}$, laser's angular frequency $\omega_{L}$, electron mass $m_{e}$, and speed of light $c$. However, the pump's spatiotemporal intensity distribution in plasma will differ depending on the plasma's density distribution, the pump's focusing location and its propagation distance into the plasma. The plasma originated from helium gas with 5\% nitrogen dopant contained in a gas cell and ionized by the pump's rising edge [27].

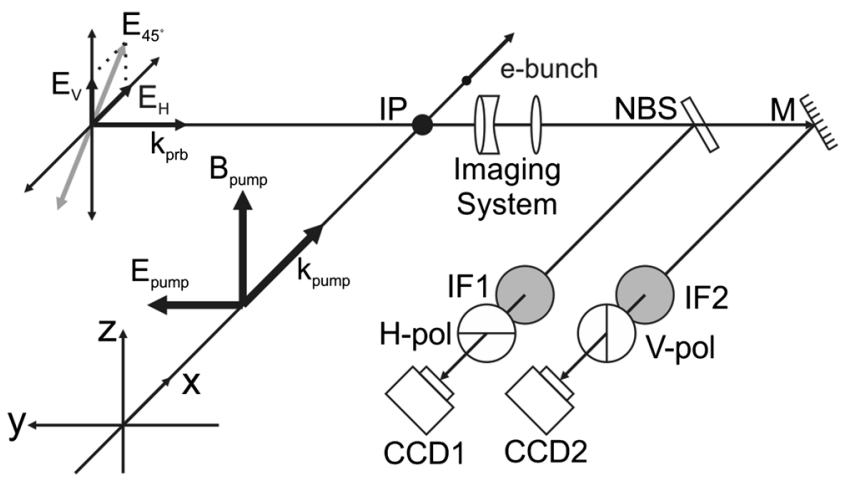

FIG. 1. Experimental setup.

By measuring the plasma wave's periodic structure from the shadowgrams [17], an average plasma wavelength of $8.6 \mu \mathrm{m}$ was measured, corresponding to an on-axis background electron plasma density $n_{e} / \gamma$ of $(1.5 \pm 0.1) \times$ $10^{19} \mathrm{~cm}^{-3}$ with $\gamma=1$ being the Lorentz factor for plasma electrons $>30 \mu \mathrm{m}$ behind the driver.

As seen in Fig. 1, the pump (probe) propagates in $x$ direction ( $-y$ direction). The probe [28] is linearly polarized at $45^{\circ}$ to the $x$ axis in the $x z$ plane and illuminates the interaction point into which the linearly polarized pump is focused. The accelerated electron bunches (e-bunch) are measured in a dipole spectrometer (not shown). Using a VIS-NIR corrected imaging system, nonpolarizing beam splitter (NBS) and a silver coated mirror (M), the $x z$ plane of the pump-plasma interaction is imaged simultaneously onto two CCDs. Polarizers (H-pol, V-pol) and various interference filters (IF1, IF2) could be used to simultaneously image the interaction driven by a single shot of the pump laser using two different polarization orientations and/or spectral ranges of the probe. Horizontal polarization of the probe (H-pol) refers to linear polarization along the $x$ direction while vertical polarization $(\mathrm{V}-\mathrm{pol})$ is along the $z$ direction (see $E_{H}$ and $E_{V}$ in Fig. 1). Shadowgrams of the laser-plasma interaction were recorded over approximately $2 \mathrm{~mm}$ of propagation by delaying the probe with respect to the pump and shifting the imaging system along the $x$ axis when necessary. The very beginning and end of the interaction region were blocked to the imaging system by the entrance and exit walls of the gas cell. The FWHM pulse duration of the probe was shown to run reliably at $\leq 4$ fs with a broadband spectrum ranging from 500 to $950 \mathrm{~nm}[29]$.

Due to the variability in shot-to-shot performance of the LWFA setup, the recorded shadowgrams were sorted based on the measured characteristics of the accelerated electron bunch. Following the simplifying assumption that similar characteristics of the electron spectra obtained in different pump shots indicate similar laser-plasma interactions enables more dependable comparison between shadowgrams taken with different shots but otherwise near constant pump and plasma parameters. This allows not only for comparison of 
shadowgrams taken at different propagation distances of the pump, but also for comparison of different shadowgrams recorded with different probe polarizations and spectral ranges that could not be simultaneously recorded.

\section{EXPERIMENTAL RESULTS}

Our investigation of the pump during its propagation through the plasma using RECRs is based on two experimental data sets. The first set employs the spectral dependence of RECRs as detected by the probe, while the second set shows explicitly a result of the RECR's polarization dependence. In the first data set, only the probe's H-pol state was recorded; however, different interference filters were used to investigate the RECR's frequency dependence. Figure 2 displays exemplary shadowgrams from this data set at five locations spaced throughout the pump's propagation using $40 \mathrm{~nm}$ FWHM bandpass filters centered at $800 \mathrm{~nm}$ [(a)-(e)] and $660 \mathrm{~nm}$ [(f)-(j)]. Shadowgrams centered at $850 \mathrm{~nm}$ were also recorded but are omitted here for reasons discussed shortly. The pump always travels from left to right and its peak is positioned just right of center in each image.

Two features visualized by RECRs can be identified in Fig. 2. First, the region at the front of the wakefield structure overlapping with the pump's peak intensity changes its shape and intensity modulation with increasing propagation distance of the pump and when using different bandpass filters with the probe. This will be called the asymmetric signal, for reasons which will be clarified later. Images (f) and (g) recorded at $660 \mathrm{~nm}$ show a "teardrop" shaped feature while (a) and (b) recorded at $800 \mathrm{~nm}$ exhibit a diffuse scattering and often only a single dark/bright spot on axis where the peak of the laser is located. Images (c)(e) and (h)-(j) show rounder features around the pump's position including a striking difference in signal between images taken at the final recorded position (e) and (j). The second signal of interest is diffraction rings, called the halfring signal, seen clearly at position $1190 \mu \mathrm{m}$ [Figs. 2(d) and 2(i)]. They appear ahead of the pump's position after a certain propagation distance regardless of the filtered probe's spectrum.

The shadowgrams recorded at 800 and $850 \mathrm{~nm}$ showed very similar results because of how the electron-cyclotron frequency $\Omega_{\text {ce }}$ (henceforth simply "cyclotron frequency") changes with the local magnetic field of the pump laser. By investigating the relationship between the laser's intensity, described by its normalized vector potential, and the cyclotron frequency, one can determine which spectral range of a probe pulse will overlap with the cyclotron frequency and thus experience a strong birefringence due to the pump's intensity distribution in the plasma. Note that for relativistic pump intensities $a_{0} \geq 1$, and for underdense plasma, i.e., $n_{e} \ll n_{c}$ where $n_{c}=$ $\omega_{\mathrm{prb}}^{2} m_{e} \varepsilon_{0} / e^{2}$ is the critical density (with the probe's angular frequency $\omega_{\text {prb }}$ and the permittivity of vacuum $\varepsilon_{0}$ ), it is well justified to approximate the resonance frequency of the plasma with the cyclotron frequency. With the above assumptions, this approximation is good to within a few $\mathrm{nm}$ of wavelength in the VIS-NIR spectral range. For higher electron plasma densities, however, the contribution of the electron plasma frequency $\omega_{\mathrm{pe}}=\sqrt{n_{e} e^{2} / m_{e} \varepsilon_{0}}$ to the relevant resonance frequencies, see [30,31], will become non-negligible and must be considered.

The normalized vector potential $a_{0}$ of a laser pulse can be converted into a magnetic field via the laser's central wavelength $\lambda_{L}$ as in Eq. (1). By combining Eq. (1) with the equation for the relativistic cyclotron frequency, shown in Eq. (2), one arrives at an expression for the normalized vector potential as a function of the cyclotron wavelength $\lambda_{\text {ce }}$ as shown in Eq. (3), where wavelengths have been used in lieu of angular frequencies. The Lorentz factor in Eq. (2) is assumed in this case to be $\gamma=\sqrt{1+a_{0}^{2} / 2}$, which considers a single electron in a linearly polarized EM field:

$$
B=\frac{a_{0}}{\lambda_{L}[\mu \mathrm{m}]} 10700 \mathrm{~T}
$$

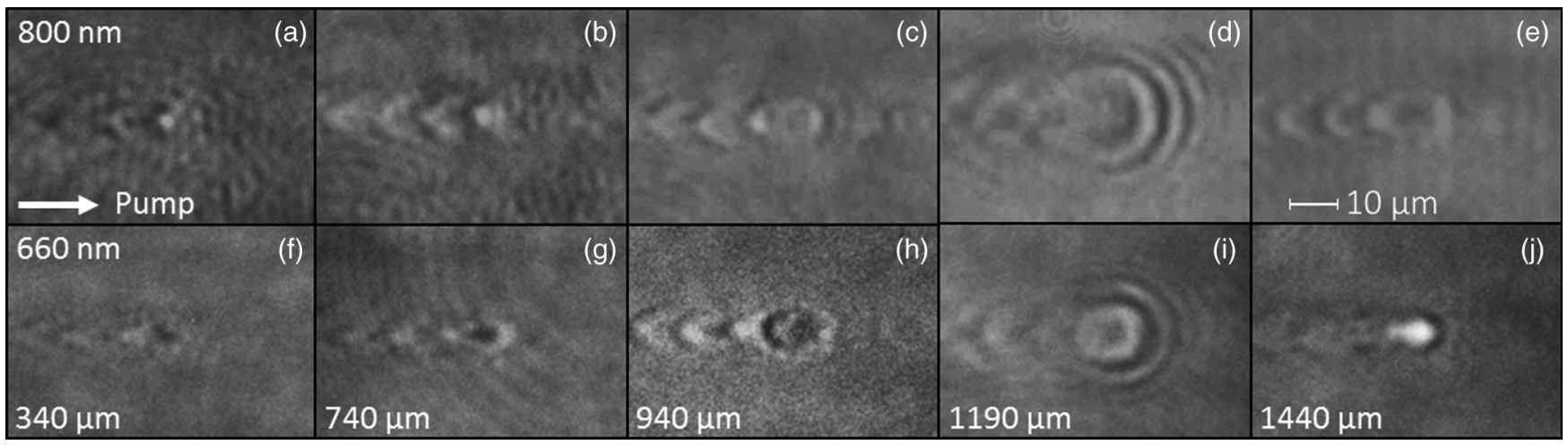

FIG. 2. From left to right, each column shows a single position along the pump's propagation. The pump's approximate position relative to the gas-cell entrance's inside edge for each image pair is given in the lower left corners of the bottom row. The H-pol probe's spectrum was filtered by $40 \mathrm{~nm}$ FWHM bandpass filters centered at $800 \mathrm{~nm}$ (a)-(e) and $660 \mathrm{~nm}$ (f)-(j). 


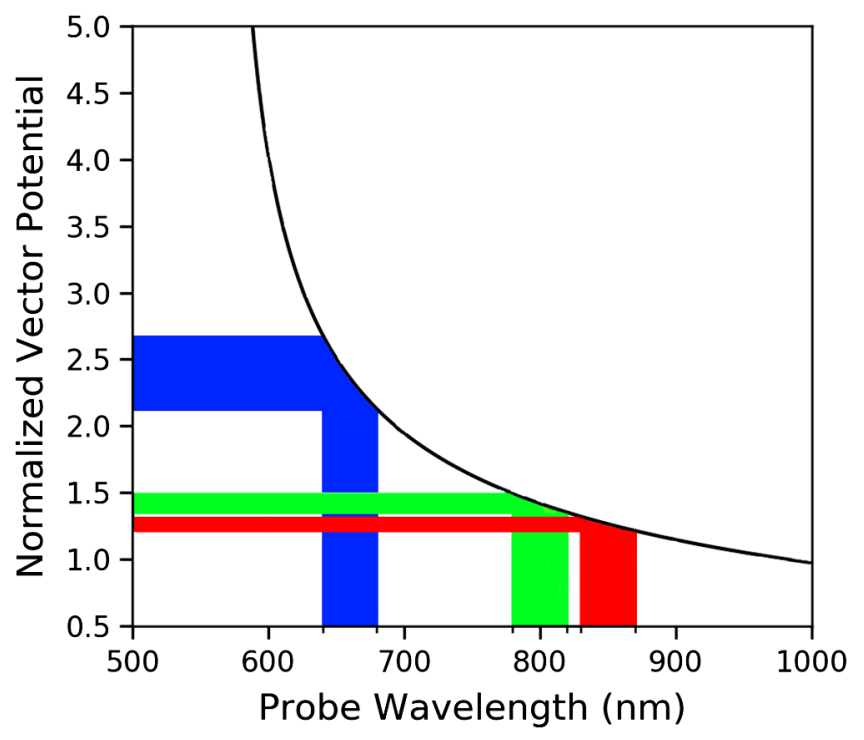

FIG. 3. Spectral dependence of the cyclotron resonance for relativistic pump intensities $\left(\lambda_{L}=800 \mathrm{~nm}\right)$ in underdense plasma. The three colored regions correspond to the three bandpass filters used in the experiment.

$$
\begin{gathered}
\Omega_{c e}=\frac{e B}{\gamma m_{e}} \\
a_{0}=\frac{\sqrt{2} \lambda_{L}}{\sqrt{2 \lambda_{c e}^{2}-\lambda_{L}^{2}}} .
\end{gathered}
$$

Figure 3 shows Eq. (3) plotted for a pump wavelength $\lambda_{L}$ of $800 \mathrm{~nm}$ over the spectral range of 500 to $1000 \mathrm{~nm}$. Since we are looking for probe wavelengths that equal the cyclotron wavelength the $x$ axis has been simply labeled "Probe Wavelength." The three colored regions, blue, green, and red, represent the three bandpass filters used in the experiment, $(660 \pm 20),(800 \pm 20)$ and $(850 \pm$ 20) $\mathrm{nm}$ respectively. One can now see why the shadowgrams recorded in the $(850 \pm 20) \mathrm{nm}$ range were omitted from Fig. 2. Each spectral region of the probe will experience cyclotron resonances at different pump intensities, i.e., at different $a_{0}$. However, the resonances of the 800 and $850 \mathrm{~nm}$ spectral regions of the probe are not separated by a significant amount as to cause visible differences in their respective shadowgrams that could be clearly presented. This is corroborated by the experimental shadowgrams, in which clear differences could be seen between the probe images filtered at 660 and $800 \mathrm{~nm}$ while only weak differences were seen between those filtered at 800 and $850 \mathrm{~nm}$. Note that Fig. 3 neglects the modifications to the resonance conditions described in the relativistic Appleton-Hartree (RAH) equation that depend on the relativistic drift motion of the plasma electrons as will be detailed in the derivation of the RAH equation shortly.

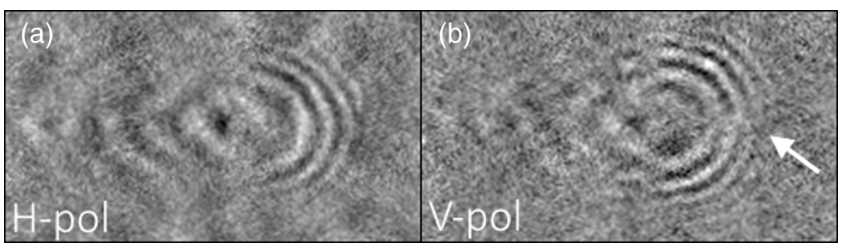

FIG. 4. H-pol (a) and V-pol (b) shadowgrams recorded with the probe's full spectrum after $1190 \mu \mathrm{m}$ propagation of the pump. Both images have been processed for improved contrast. The white arrow indicates the location of the on-axis break in the halfring signal.

A second set of data was recorded using the few-cycle probe's full spectrum but with two different orthogonal polarization states (H-pol and V-pol). Again, the half-ring signal is seen; however, as shown in Fig. 4 there is a clear difference between the diffraction rings in the H-pol and V-pol shadowgrams. The half-ring signal exhibits a break along the pump's propagation axis when recorded with the V-pol state [cf. Fig. 4(b)] but are continuous when recorded with the H-pol state [cf. Fig. 4(a)].

\section{THEORETICAL MODEL}

RECR and its effect on shadowgraphic imaging can be understood by modeling the area around the pump as cold, collisionless plasma magnetized by the pump, while also accounting for the plasma electrons' relativistic drift motion moving under the influence of the pump and surrounding plasma distribution. The pump's cycle-averaged magnetic field is used in lieu of a strictly static magnetic field. Starting with the collisionless AppletonHartree (AH) equation from magnetoionic theory [30,31], the refractive indices of magnetized plasma can be analytically described. The plasma electrons' motion is separated into the drift motion of their guiding centers and the residual motion around those centers which resembles cyclotron gyration or the well-known figure-of-eight trajectory [32]. The $\mathrm{AH}$ equation is then rewritten in the frame of each guiding center requiring the full Lorentz transformation of the pump, probe and local plasma's parameters into said frame(s). The resulting relativistic Appleton-Hartree (RAH) equation must then be numerically solved for the plasma's refractive indices in the lab frame.

The modification of the $\mathrm{AH}$ equation to account for the relativistic motion of the plasma electrons builds on work reported in [33]. The formalism used here will allow motion of the plasma in any direction as well as take into account all electric and magnetic field components from the pump and plasma. We first denote quantities associated with the lab frame using unprimed variables and those associated with the relativistic drift frame of the plasma electrons' guiding centers with primed variables. The collisionless $\mathrm{AH}$ equation is then written in the drift frame of a plasma electron as given in Eq. (4) with $\eta_{ \pm}^{\prime}$ being the refractive 
indices, $k^{\prime}$ the probe's wave number, $\omega^{\prime}$ the probe's angular frequency, $\omega_{\mathrm{pe}}^{\prime}$ the electron-plasma frequency, $\Omega_{\mathrm{ce}}^{\prime}$ the electron-cyclotron frequency, and $\theta_{\mathrm{kB}}^{\prime}$ the angle between the probe's propagation vector $\vec{k}^{\prime}$ and the local magnetic field vector $\vec{B}^{\prime}$. The drift frame of interest for each plasma electron is the drift frame of its guiding center in which figure-of-eight motion can be observed while under the influence of the pump laser:

$$
\begin{aligned}
\eta_{ \pm}^{\prime 2} & =\frac{c^{2} k^{\prime 2}}{\omega^{\prime 2}} \\
& =1-\frac{\left(\frac{\omega_{\mathrm{pe}}^{\prime}}{\omega^{\prime}}\right)^{2}}{1-\frac{\left(\frac{\Omega_{\mathrm{ce}}^{\prime}}{\omega^{\prime}}\right)^{2} \sin ^{2} \theta_{k B}^{\prime}}{2\left[1-\left(\frac{\omega_{\mathrm{pe}}^{\prime}}{\omega^{\prime}}\right)^{2}\right]} \pm \sqrt{\frac{\left(\frac{\Omega_{\mathrm{ce}}^{\prime}}{\omega^{\prime}}\right)^{4} \sin ^{4} \theta_{\mathrm{kB}}^{\prime}}{4\left[1-\left(\frac{\omega_{\mathrm{pep}}^{\prime}}{\omega^{\prime}}\right)^{2}\right]^{2}}+\left(\frac{\Omega_{\mathrm{ce}}^{\prime}}{\omega^{\prime}}\right)^{2} \cos ^{2} \theta_{\mathrm{kB}}^{\prime}}} .
\end{aligned}
$$

By defining the drift trajectory of each plasma electron's guiding center $\vec{v}$ in Cartesian coordinates and the corresponding Lorentz factor $\gamma_{D}=1 / \sqrt{1-v^{2} / c^{2}}$ one can use the full Lorentz transformation in Cartesian coordinates [34] to define the probe's angular frequency and propagation vector in the drift frame as functions of its angular frequency and propagation vector in the lab frame and the drift frame's trajectory in the lab frame. As shown in the experimental setup described in Fig. 1 the probe propagates along the $-y$ axis in the lab frame. From this geometry Eq. (5) shows the probe's angular frequency in the drift frame depending on the lab frame's refractive indices $\eta_{ \pm}$, drift frame's Lorentz factor $\gamma_{D}$, and the drift frame's $y$-component velocity relative to the lab frame $v_{y}$. Similarly, the probe's propagation vector in the drift frame is described by Eq. (6). In both Eqs. (5) and (6), the labframe wave number of the probe has been replaced using $k_{y}=-\omega \eta_{ \pm} / c$ :

$$
\begin{gathered}
\omega^{\prime}=\omega \gamma_{D}\left(1+\eta_{ \pm} \frac{v_{y}}{c}\right) \\
\vec{k}^{\prime}=\left(\begin{array}{c}
\frac{-\omega}{c} \gamma_{D} \frac{v_{x}}{c}-\eta_{ \pm} \frac{\omega}{c}\left(\gamma_{D}-1\right) \frac{v_{x} v_{y}}{v^{2}} \\
\frac{-\omega}{c} \gamma_{D} \frac{v_{y}}{c}-\eta_{ \pm} \frac{\omega}{c}\left(1+\left(\gamma_{D}-1\right) \frac{v_{y}^{2}}{v^{2}}\right) \\
-\frac{\omega}{c} \gamma_{D} \frac{v_{z}}{c}-\eta_{ \pm} \frac{\omega}{c}\left(\gamma_{D}-1\right) \frac{v_{y} v_{z}}{v^{2}}
\end{array}\right) .
\end{gathered}
$$

As we are interested in the plasma magnetized by the pump pulse ahead of the wakefield structure, we can use the simplifying assumption that the electric and magnetic fields of the pump dominate in this area. This assumption is justified when using relativistically intense pump pulses in underdense plasma as simulations show the fields associated with the wakefield or plasma at the location of the pump to be at least a factor of 100 weaker, meaning their respective cyclotron resonances lay significantly outside of the VIS-NIR spectral range of the probe used in the current experiments. Again, using the full Lorentz transformation of the pump's electric $\vec{E}$ and magnetic $\vec{B}$ fields in Cartesian coordinates one can arrive at an expression for the magnetic field of the pump in the drift frame of the plasma electrons shown in Eq. (7). By using Eqs. (6) and (7), one can now easily calculate $\theta_{k B}^{\prime}$ :

$$
\vec{B}^{\prime}=\left(\begin{array}{l}
\gamma_{D} B_{x}-\frac{\gamma_{D}}{c^{2}}\left(v_{y} E_{z}-v_{z} E_{y}\right)-\frac{\left(\gamma_{D}-1\right) v_{x}}{v^{2}}(\vec{v} \cdot \vec{B}) \\
\gamma_{D} B_{y}-\frac{\gamma_{D}}{c^{2}}\left(v_{z} E_{x}-v_{x} E_{z}\right)-\frac{\left(\gamma_{D}-1\right) v_{y}}{v^{2}}(\vec{v} \cdot \vec{B}) \\
\gamma_{D} B_{z}-\frac{\gamma_{D}}{c^{2}}\left(v_{x} E_{y}-v_{y} E_{x}\right)-\frac{\left(\gamma_{D}-1\right) v_{z}}{v^{2}}(\vec{v} \cdot \vec{B})
\end{array}\right) .
$$

The electron plasma frequency $\omega_{\text {pe }}^{\prime}$, Eq. (8), exhibits a Lorentz invariant quantity $n_{e} /\left(\gamma_{D} m_{e}\right)$ which from the lab frame can be understood as the "relativistic mass increase" of the electron, and from the drift frame can be understood as the relativistic contraction of the volume element used to define the electron plasma density $n_{e}[35]$ :

$$
\omega_{\mathrm{pe}}^{\prime}=\sqrt{\frac{n_{e} e^{2}}{\varepsilon_{0} \gamma_{D} m_{e}}} .
$$

Finally, the electron-cyclotron frequency $\Omega_{\mathrm{ce}}^{\prime}$, Eq. (9), is defined using the Lorentz transformed, cycle-averaged magnetic field of the pump $B^{\prime}$ and the residual electron velocity in the drift frame, here defined with the Lorentz factor $\gamma_{Q}^{\prime}$. The use of cycle-averaged values for $B^{\prime}$ and $\gamma_{Q}^{\prime}$ approximates calculating an effective relativistic cyclotron frequency for the electrons' figure-of-eight motion around their guiding centers. As previously described, the labframe trajectory of each plasma electron can be described as the drift motion of the electron's guiding center, and a residual motion of the electron relative to its guiding center, in this case the figure-of-eight motion. $\gamma_{Q}^{\prime}$ is associated with the electrons' figure-of-eight velocity as seen from the drift frames of their guiding centers. By substituting Eqs. (5)-(9) into Eq. (4) one arrives at a version of the RAH equation that can be numerically solved for the lab frame's refractive indices $\eta_{ \pm}$. The process of numerically solving the RAH equation using LWFA simulation data is given in Sec. VI:

$$
\Omega_{\mathrm{ce}}^{\prime}=\frac{e B^{\prime}}{\gamma_{Q}^{\prime} m_{e}}
$$

Both the $\mathrm{AH}$ and the RAH equations predict resonance and reflection points at several characteristic frequencies of an EM wave propagating through the plasma. In underdense plasma magnetized by a relativistically intense pump laser, and assuming static ions, the characteristic frequency of interest is the local electron-cyclotron frequency, given here simplified to its nonrelativistic form of $\Omega_{\mathrm{ce}}=e B / m_{e}$ with the local magnetic field $B$. The magnetic field $B=$ 
$a_{0} \lambda_{L}^{-1}[\mu \mathrm{m}] \times 10.7 \mathrm{kT}$ of a relativistically intense pump centered at $800 \mathrm{~nm}$ and with an $a_{0}$ in the range of 0.8 to 2.0 is in the tens of kilotesla range. The corresponding cyclotron wavelengths of such a magnetic field lie in the VIS-NIR spectral range of 400-1000 nm. Any probe with a spectrum near the cyclotron frequency and a polarization component parallel to the plane of cyclotron motion encounters resonance and reflection points and is thus directly sensitive to the pump's magnetic field. Polarization components perpendicular to the plane of cyclotron motion effectively see unmagnetized plasma.

For the resonant wave, the resonance points, i.e., refractive index $\eta \rightarrow \infty$, are associated with absorption of the wave in plasma (cf. electron-cyclotron resonant heating $[36,37])$, while the reflection points, i.e., $\eta \rightarrow 0$, result in an evanescent wave mode and reflection of the wave (cf. plasma mirror [38]). However, for the present case, strong absorption and reflection of the probe are not expected. At the subpicosecond timescale presently considered, the electron-electron and electron-ion collision rates are not effective in converting electron motion due to the probe into thermal motion, i.e., absorption of the probe by the plasma. Furthermore, the few- to submicrometer extent of local plasma that exhibits reflection behavior is also not effective in fully reflecting the EM wave. Thus, both resonance and reflection behaviors are henceforth referred to as anomalous dispersion $(\eta \not 1)$, as their effect on shadowgraphic imaging manifests itself primarily via strong refraction of the probe relative to nonmagnetized, tenuous plasma and not via absorption and reflection.

\section{3D ЕРОСН PARTICLE-IN-CELL SIMULATION AND RESULTS}

The RAH equation captures the essential aspects of the plasma's spectrally dependent birefringence near the pump's peak intensity. However, considering the system's complexity (i.e., comoving pump and transverse probe, few-cycle microscopy and relativistic plasma motion) particle-in-cell (PIC) simulations helped to further understand the origins and appearance of the signals seen in the recorded shadowgrams. Details on using the EPOCH code [39] to create simulated shadowgrams can be found in [40].

The RECR signals recorded in the experiment are also seen in simulated shadowgrams using data from the EPOCH simulation. As seen in Fig. 14 of [40], the simulation results for the spectrally filtered shadowgrams produced with an $\mathrm{H}$-pol probe also show a drastic change in the front of the plasma wave for spectral ranges of $(600 \pm 10),(700 \pm 10)$, $(800 \pm 10)$ and $(900 \pm 10) \mathrm{nm}$, while the trailing plasma wave itself shows significantly less change in its appearance with changing probe spectrum. To investigate this signal Fig. 5 was created using the EPOCH simulation data. The phase map in yellow/green is calculated from the probe having been self-consistently propagated using the PIC algorithm (for details cf. [40]). The plotted phase has been accumulated by the probe over $\sim 2$ fs of propagation and is relative to the phase it would have accumulated via similar propagation through unperturbed, unmagnetized plasma. For different snapshots of the simulation, a simulated shadowgram [cf. Figs. 5(g)-5(i)] is also generated taking into account only the probe's accumulated phase up to its current respective position. Note that Figs. 5(a)-5(f) show
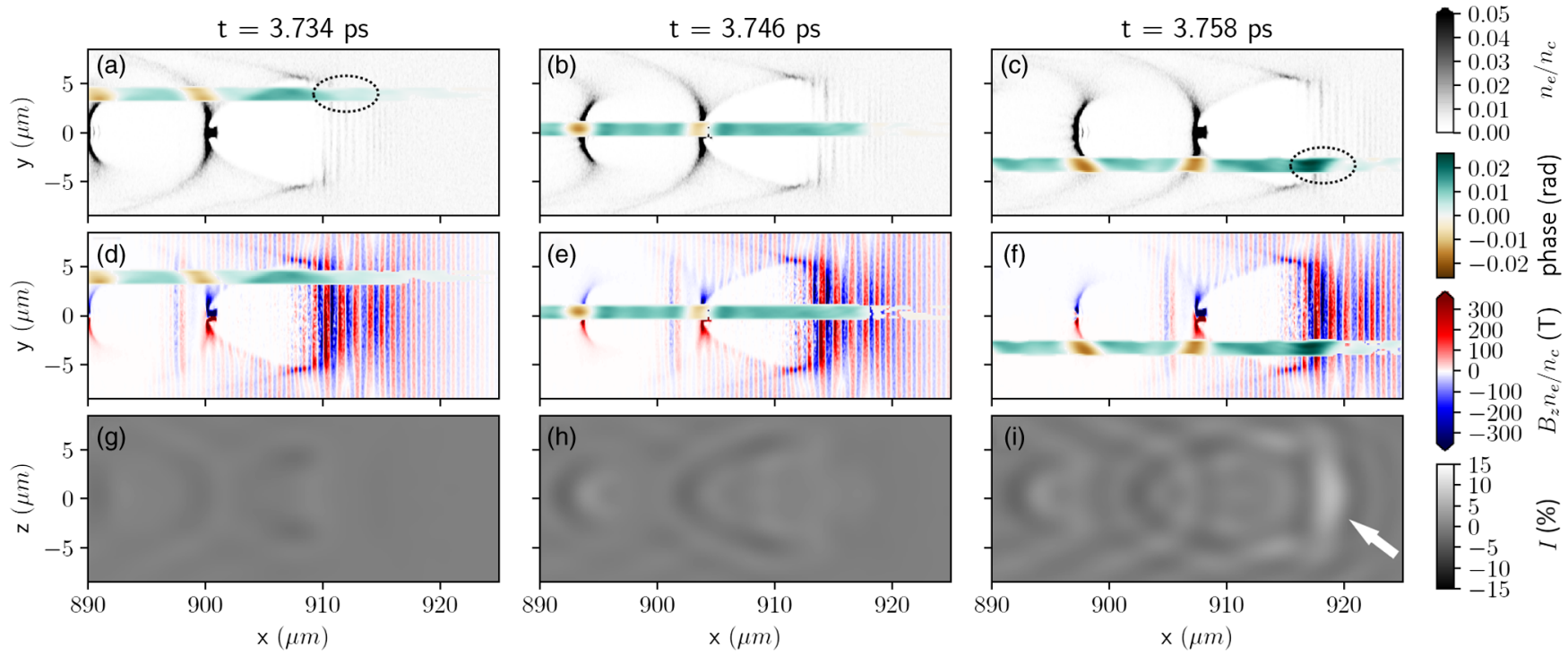

FIG. 5. (a)-(c) Normalized plasma density with overlay of probe's relative phase in radians. (d)-(f) Scaled magnetic fields with phase overlay. (g)-(i) Relative irradiance modulation of simulated shadowgram in \%. The H-pol probe propagates in the $-y$ direction and its FWHM temporal extent is coincident with the green/yellow phase colormap. Shadowgrams (g)-(i) are created using only the phase accumulated up to the indicated probe position. Plots (a)-(f) correspond to xy planes at $z=0 \mu \mathrm{m}$. The white arrow denotes strong brightness modulation described in the text. 
the $x y$ plane in the plasma at $z=0 \mu \mathrm{m}$, while Figs. 5(g)-5(i) show the resulting shadowgram using the full $3 \mathrm{D}$ plasma distribution and respective propagation distances of the probe.

The strong brightness modulation at the front of the wakefield in the shadowgram [cf. Fig. 5(i), white arrow] first appears after the probe has crossed the pump's optical axis ( $x$ axis). The accumulated phase map clearly shows the asymmetric signal's origin: while there is a pronounced phase signal around $x=917 \mu \mathrm{m}$ for a probe position of $y=-4 \mu \mathrm{m}$ [cf. Fig. 5(c), dotted ellipse], there is no such phase signal for a probe position of $y=+4 \mu \mathrm{m}$ [cf. Fig. 5(a), dotted ellipse]. The irradiance modulation in the shadowgram [cf. white arrow in Fig. 5(i)] arises from phase in the probe beam accumulated in a region of plasma in $-y$ space overlapping with the pump, even though the same general conditions, e.g., pump's magnetic field amplitude and plasma density, exist at the symmetric position in $+y$ space. The only difference is the direction of the plasma electrons' relativistic drift velocities and the resulting relativistic Doppler shifts of the probe's spectrum in these drift frames. The source of the asymmetric signal was corroborated by combining particle- and field-tracking data from 2D PIC simulations using vsim and the RAH equation. As will be demonstrated in Sec. VI, it was found that for increasing drift velocity of the plasma electrons, i.e., higher pump laser intensities, the accumulated phase of interest becomes more and more localized to the $-y$ space.

The second RECR signal seen in both the experimental and simulated shadowgrams is the half-ring signal ahead of the pump's position. These diffraction rings are visible in Figs. 6(d) and 13(a) of [40] as well as Fig. 3(b) of [17] for a pump propagation in the latter case of $v_{g} t \geq 913 \mu \mathrm{m}$. This feature can be understood as a manifestation of RECR in the context of a self-steepening pump. At the beginning of the pump's propagation in the plasma its 27 fs FWHM duration and $\sim 10 \mu \mathrm{m}$ spot size are mismatched to the plasma density having a plasma wavelength of $8.6 \mu \mathrm{m}$. No diffraction rings are seen because the locations of anomalous dispersion at each probe frequency are spatially spread across the pump's relatively broad intensity distribution. As the pump propagates through the plasma it self-steepens [41,42]. The pump's steeper intensity gradient causes the anomalous dispersion for a broader range of probe frequencies to be extremely localized, akin to a step function change in refractive index from which the probe diffracts. As with the plasma wave's spatial period, the diffraction rings are only resolved using a few-cycle probe, otherwise the copropagating nature of the diffraction rings and a $>10 \mathrm{fs}$ duration probe would temporally average out the rings' irradiance modulation [compare Figs. 13(a) and 13(b) in [40]].

By inspecting Fig. 3 of [17] one sees that this effect was already reproduced by simulation, though not yet consistently explained theoretically. The red contour lines in
Fig. 3(a) of [17] show the pump's magnetic field distribution which steepens drastically with propagation resulting in highly localized resonances and visible diffraction rings after traveling ca. $900 \mu \mathrm{m}$. The experimental shadowgrams presented in this paper, having very similar laser and plasma parameters to those in [17], also exhibit diffraction rings after ca. $900 \mu \mathrm{m}$ of propagation.

The self-consistent nature of the EPOCH simulation and the generation of simulated shadowgrams have one drawback. Although one can change the simulation's parameters to investigate how they affect the resulting shadowgram, it proves difficult to identify the physical cause of certain signals in the system. One can see the difference in the probe's accumulated phase with propagation through the plasma wave as shown in Fig. 5, but the exact physical origin of this signal remains unclear. To delve further into what causes the asymmetric and half-ring signals, 2D PIC simulations using the vsim code to produce particleand field-tracking data were carried out and analyzed as described in the following section.

\section{2D vSim PARTICLE-IN-CELL SIMULATIONS AND RESULTS}

To test the results of the RAH equation against the signals seen in the experimentally recorded shadowgrams and in the simulated shadowgrams produced using the EPOCH simulation we further ran 2D simulations using the PIC code vsim [43]. The advantage of using the vsim code is its built-in particle- and field-tracking capabilities which can be used to store each macroparticle's (i.e., electron's) position, velocity four-vector including its velocity out of the simulation plane, the $E$ and $B$ fields it encounters as well as the local plasma density for every time step of the simulation. Using this data, the drift frame of each macroparticle's guiding center could be calculated and the resulting lab-frame refractive indices calculated via the RAH equation were then compared to the results of the EPOCH simulation and the experimental data.

There is an underlying assumption in calculating a "bulk" parameter such as a refractive index from individual particle data such as that recorded using the macroparticles. This assumption is that discrete macroparticles in the vsim simulation represent a plasma fluid such that the calculated refractive index using data from a single macroparticle is valid for the local region around that macroparticle. We took this assumption to be valid only for locations and times in the vsim simulation where the tracked macroparticles' trajectories do not cross. Trajectory crossings of the macroparticles in a PIC simulation of LWFA are typically first seen after the macroparticles have already moved behind the pump pulse and begin interacting with the fields of the plasma wave [44]. Said trajectory crossing would produce, for the same local plasma, two different drift velocities resulting in an ambiguity as to which drift 


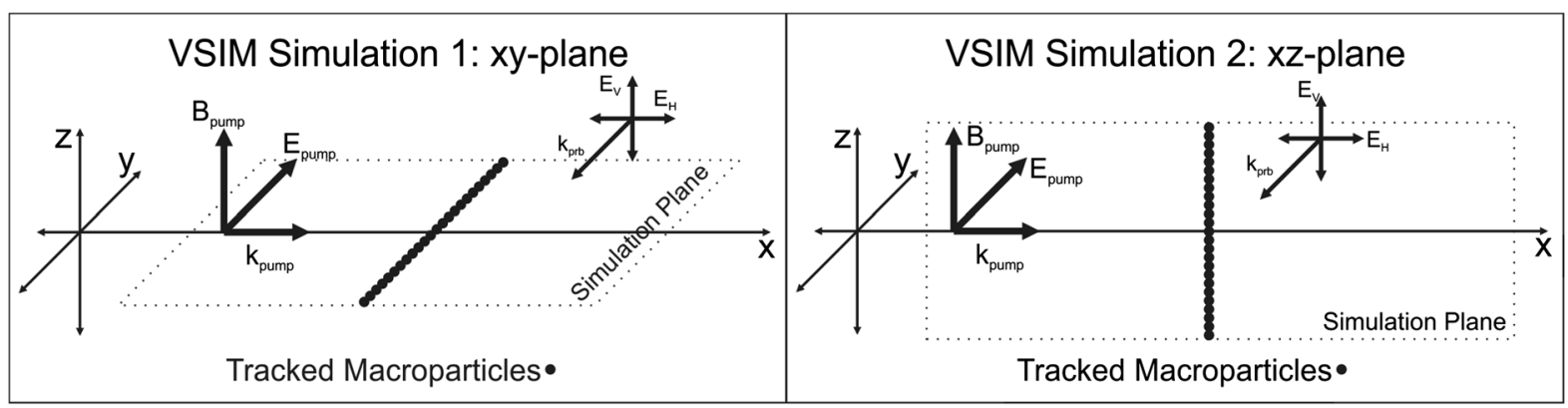

FIG. 6. Setup of the two 2D vsim simulations.

velocity is used to calculate the local plasma's refractive indices.

2D simulations with vsim were used in order to investigate the RECR signals in the vicinity of the pump laser in the $x y$ and $x z$ planes (refer to Fig. 1 for system's coordinates). In each simulation, the data from a line of macroparticles (see Fig. 6) were tracked. The probe pulse was not present in the simulations but could be mathematically represented during processing of the data using the $\mathrm{RAH}$ equation. The simulations were set up as follows. The simulation boxes had 4850 cells with $25 \mathrm{~nm}$ resolution in the longitudinal direction and 420 cells with $100 \mathrm{~nm}$ resolution in the transverse direction. The pump laser with a center wavelength of $800 \mathrm{~nm}$ was focused to the middle of the simulation box to a FWHM spot size of $10 \mu \mathrm{m}$. Each simulation ran for 5220 temporal steps over a total duration of roughly $420 \mathrm{fs}$, but the data from the particle and field tracking used with the RAH equation was only recorded over 999 temporal steps ( 80 fs) during which the pump pulse overtook the line of macroparticles. Each simulation was run twice (total of four simulations), once with a pump pulse FWHM duration of 15.7 fs and an $a_{0}$ of 3.5, and again with FWHM duration of 6 fs and $a_{0}$ of 5.0. The latter parameters match more accurately the parameters of the
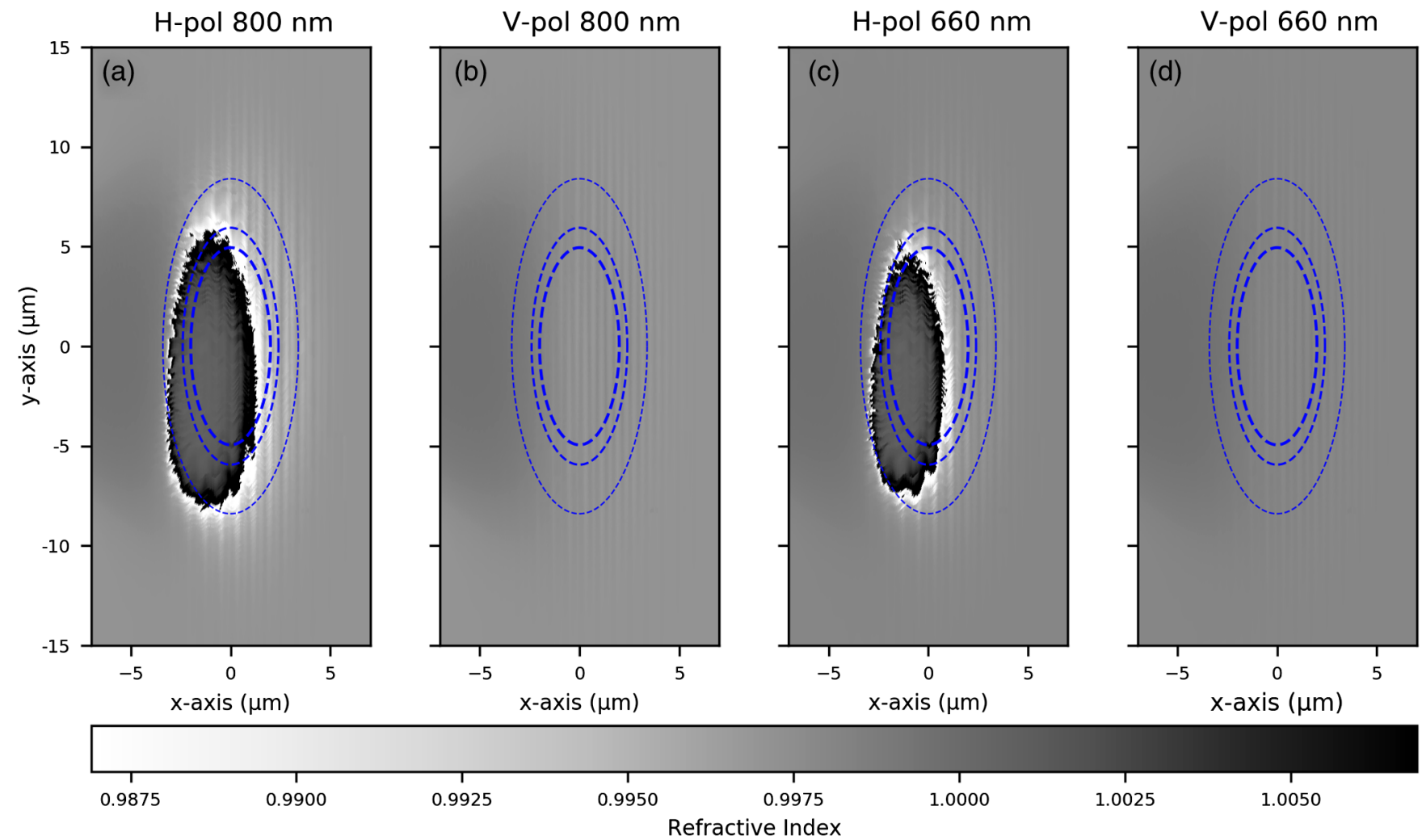

FIG. 7. Refractive index of the plasma in the $x y$ plane for different probe polarizations and wavelengths induced by a pump pulse with $15.7 \mathrm{fs}$ FWHM duration and an $\boldsymbol{a}_{0}$ of 3.5. The blue, dashed lines represent the FWHM, 1/e and 1/e $\mathrm{e}^{2}$ intensity contours of the Gaussian pump pulse. Each subplot of the figure is labeled with the probe beam's polarization (H-pol or V-pol) and its wavelength (800 or $660 \mathrm{~nm})$. The figures are calculated from data produced from vsim in simulating the $x y$ plane. 


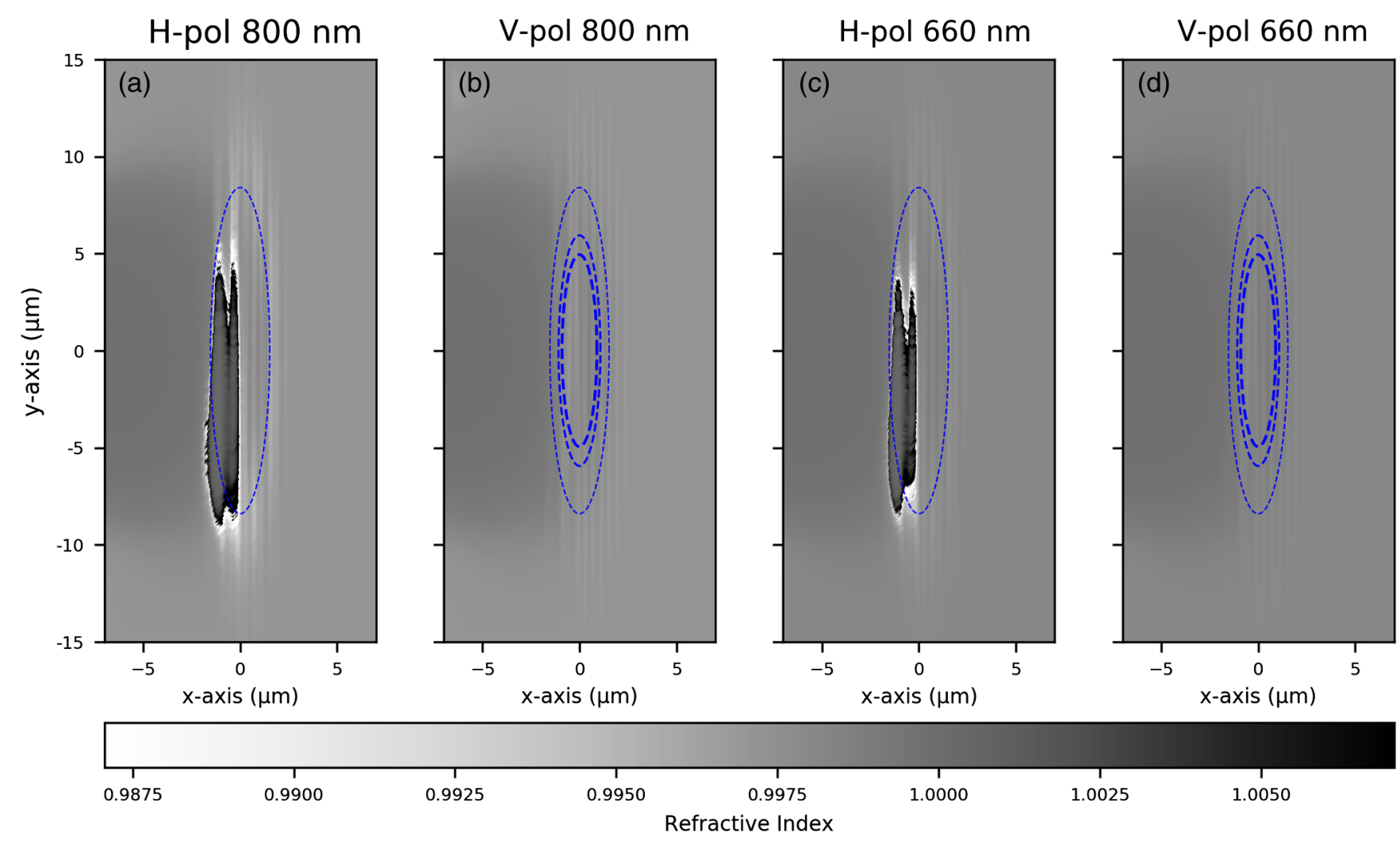

FIG. 8. Refractive index of the plasma in the $x y$ plane for different probe polarizations and wavelengths induced by a pump pulse with a 6.0 fs FWHM duration and an $\boldsymbol{a}_{0}$ of 5.0. The blue, dashed lines represent the FWHM, 1/e and 1/ $\mathrm{e}^{2}$ intensity contours of the Gaussian pump pulse. Note that in both H-pol subplots the two inner contour lines have been omitted as to not block visibility of the asymmetric signal. Each subplot of the figure is labeled with the probe beam's polarization (H-pol or V-pol) and its wavelength (800 or $660 \mathrm{~nm})$. The figures are calculated from data produced from vsim in simulating the $x y$ plane.

pump laser from the $\mathrm{EPOCH}$ simulation at a propagation distance of $913 \mu \mathrm{m}$.

Due to the short longitudinal length of the tracked simulation box, the self-evolution of the pump pulse does not need to be accounted for. This allowed during postprocessing of the data for the approximate mapping of the resulting refractive index over the entire tracked $2 \mathrm{D}$ simulation plane, described as follows. As the pump propagates through the simulation box the line of macroparticles "line scans" the parameters in the simulation plane, i.e., plasma density, particle trajectories and EM fields. Since the pump-plasma system is in a steady state, one can reconstruct recorded parameters over the whole 2D simulation plane by accounting at each time step of the simulation for the distance between the pump's peak intensity and the position of each macroparticle. These 2D parameter maps can then be used to calculate $2 \mathrm{D}$ plots of the refractive indices for comparison to experiment and to the EPOCH simulation.

Calculating the solution to the RAH equation was performed for each tracked macroparticle sequentially from the start to the end of each vsim simulation. The RAH equation is rewritten to be a polynomial of $\eta_{+}$or of $\eta_{-}$depending on the initial polarization state of the probe pulse under investigation. The zero crossings of the polynomial then give possible values of the refractive index of the plasma in the lab frame as experienced by that probe pulse. The refractive index at the start of the simulation will be that of unmagnetized plasma, easily calculable, because the fields of the pump pulse have yet to arrive at the line of macroparticles and the local plasma density is well known. By assuming that the solution to the RAH equation over time is smooth and continuous, one can track the physical solution (a zero crossing of the polynomial) with each time step of the simulation. The only complication arises when the solution to the polynomial passes through a resonance or reflection point of the RAH equation. Here, the value of refractive index can pass through 0 or move quickly towards $\infty$. Due to this discontinuous behavior and the resulting ambiguity in the physical solution of the RAH equation around the time/ location of a resonance, we use the reconstructed maps of refractive index not as an indicator of the exact refractive index in the plasma but rather as an indicator as to where anomalous dispersion and strong birefringence are to be expected.

By combining the RAH equation with the tracked macroparticle data of the vsim simulations in the $x y$ plane, 


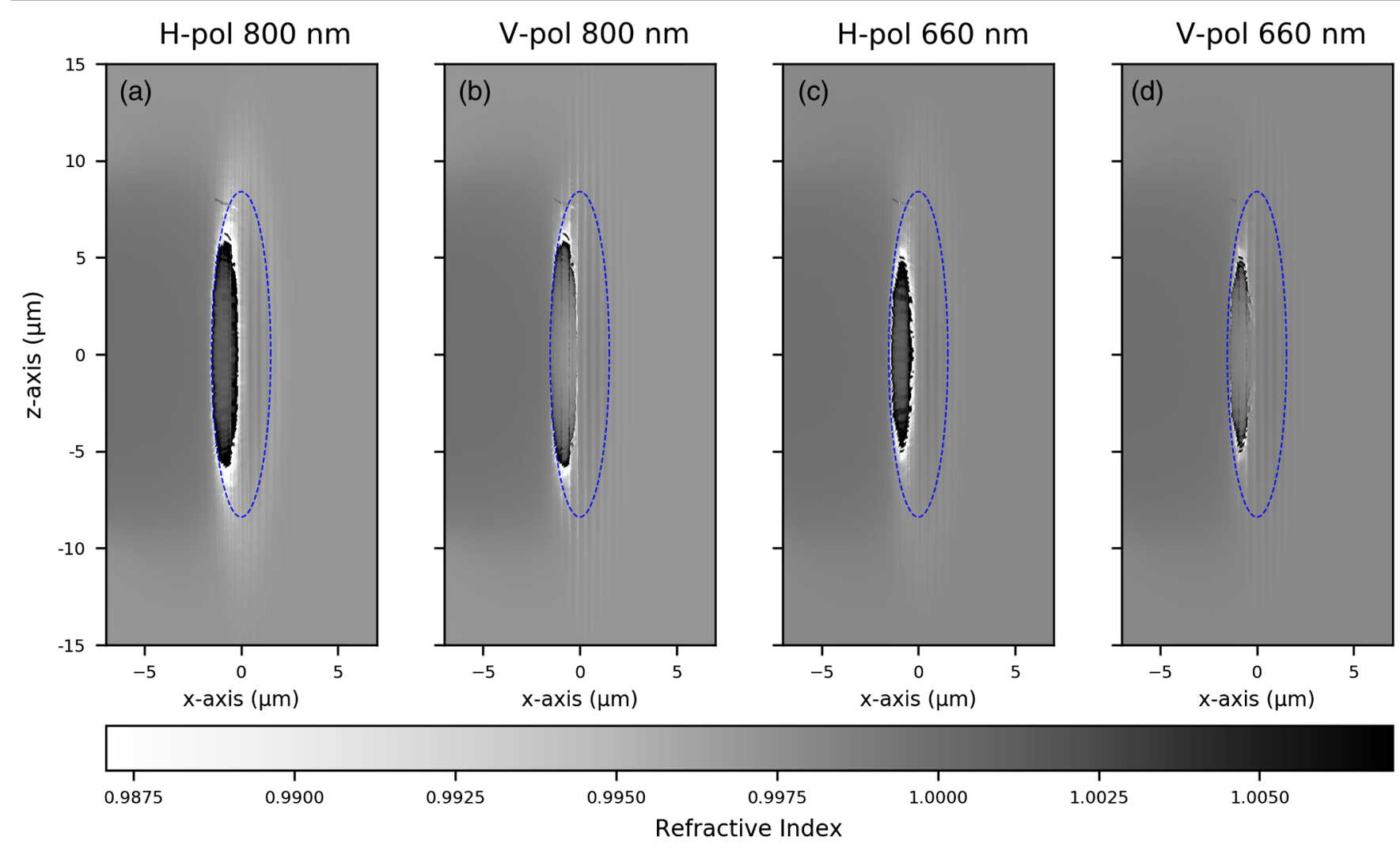

FIG. 9. Refractive index of the plasma in the $x z$ plane for different probe polarizations and wavelengths induced by a pump pulse with a 6.0 fs FWHM duration and an $\boldsymbol{a}_{0}$ of 5.0. The blue, dashed line represents the 1/ $\mathrm{e}^{2}$ intensity contour of the Gaussian pump pulse. Each subplot of the figure is labeled with the probe beam's polarization (H-pol or V-pol) and its wavelength (800 or $660 \mathrm{~nm})$. The figures are calculated from data produced from vsim in simulating the $x z$ plane.

the refractive indices in the lab frame for both H-pol and $\mathrm{V}$-pol probe pulses were calculated and mapped to the plane. The results are shown in Figs. 7 and 8 which show for the $\mathrm{H}$-pol probe a region of anomalous dispersion not seen in the results using a V-pol probe pulse. In Figs. 7(a) and 7(c) the signal is centered asymmetrically relative to the propagation axis of the pump pulse and is seen to have an elliptical shape in which the refractive index transitions quickly from a value below the normal unmagnetized refractive index to a value above the normal unmagnetized refractive index (moving from outside to inside the ellipse). As the wavelength of the probe is changed from 800 to $660 \mathrm{~nm}$, one observes that the center of the elliptical signal remains offset from the pump's propagation axis, and the signal area becomes smaller in size.

Figure 8 shows the results of increasing the pump's peak normalized vector potential to 5.0 and reducing its FWHM duration to $6.0 \mathrm{fs}$. The asymmetric signal is still only visible when using an H-pol probe and the elliptical region of anomalous dispersion again shows a reduction in size when shifting the probe's wavelength from 800 to $660 \mathrm{~nm}$ [cf. Figs. 8(a) and 8(c)]. Also of note is the cleaving of the ellipse near its top $(x \approx-0.5 \mu \mathrm{m}$, $y \approx+2.5 \mu \mathrm{m})$ and bottom $(x \approx-0.5 \mu \mathrm{m}, y \approx-8.5 \mu \mathrm{m})$. In general, an increase in the laser driver's intensity correlates with a signal more localized to the $-y$ space behind the laser driver.

The position of the asymmetric signal relative to the pump and probe pulses found using the vsim data is very similar to the signal found in the EPOCH data as shown in Fig. 5. While the fully self-consistent modeling of the plasma, pump and probe in the 3D EPOCH simulation used in creating the synthetic shadowgrams captures more accurately the physics of the LWFA system, the origin of the asymmetric signal was not directly evident. In simplifying the system via a $2 \mathrm{D}$ simulation and monochromatic probe, and by directly using the relativistic motion of the plasma electrons to assess the system, the physical origin of the asymmetric signal could be identified, namely the RECR effect.

The second strong indicator that the RAH model captures the origin of the RECR signals seen in both the experimental shadowgrams and the simulated shadowgrams from ЕРОСн is shown in Figs. 9 and 10. Figure 9 shows the refractive index in the $x z$ plane as reconstructed from the particle- and field-tracking data from vsim. One now sees a large change of refractive index in both the $\mathrm{H}$-pol and V-pol probe cases, which is the source of the diffraction rings or half-ring signal in the experimental and simulated shadowgrams for a self-steepened pump pulse. 

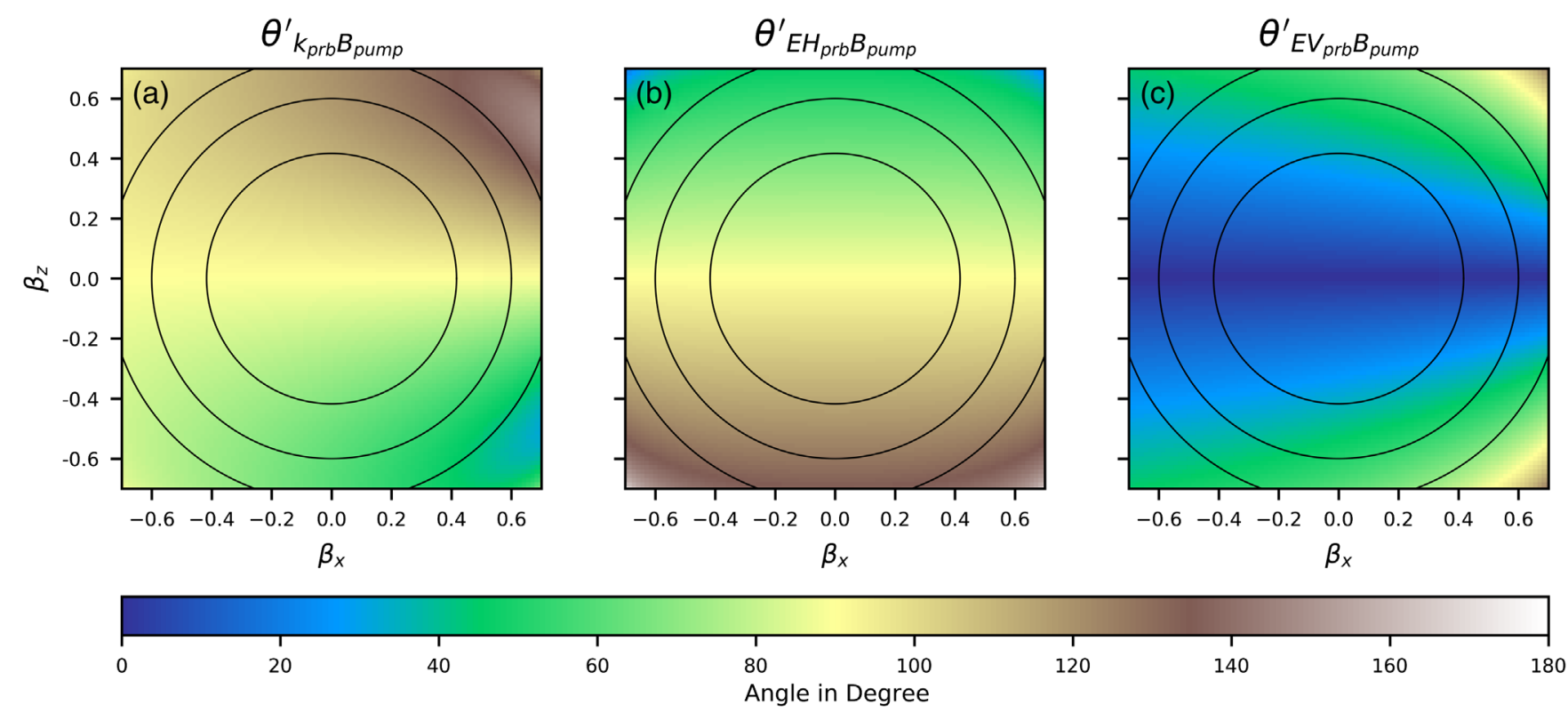

FIG. 10. Subplots show the change in vector orientation for electron drift motion in the $x z$ plane. $\boldsymbol{\beta}_{\boldsymbol{i}}=\boldsymbol{v}_{\boldsymbol{i}} / \boldsymbol{c}$ with $\boldsymbol{i}=\boldsymbol{x}, \boldsymbol{z}$. (a) Angle between the probe's propagation vector $\boldsymbol{k}_{\text {prb }}$ and the pump's magnetic field $\mathbf{B}_{\text {pump }}$. (b) Angle between the electric field of the H-pol probe $\boldsymbol{E}_{\text {prb }}$ and the pump's magnetic field $\boldsymbol{B}_{\text {pump }}$. (c) Angle between the electric field of the V-pol probe $\boldsymbol{E} \boldsymbol{V}_{\text {prb }}$ and the pump's magnetic field $\boldsymbol{B}_{\text {pump }}$. The three black, solid contour lines show the locations of the equivalent gamma factors of 1.1, 1.25, and 1.5.

It is counterintuitive that the V-pol probe also shows a drastic change in refractive index similar to that seen in the H-pol probe's results considering the V-pol probe in the lab frame is not expected to couple with the cyclotron motion of the electrons in the plasma for the given orientation of the pump's magnetic field. However, this phenomenon can be explained using Fig. 10 in which the vector orientations in the drift frame of the electron's guiding center are shown for increasing drift velocity relative to the lab frame. The angle of the probe's propagation vector to the pump's magnetic field $\theta_{k_{\mathrm{prb}} B_{\text {pump }}}^{\prime}$ [Fig. 10(a)] stays around the value of $90^{\circ} \pm$ $30^{\circ}$ for a value of the drift frame's gamma factor in the $x z$ plane up to 1.5 (outermost solid black contour line). The angle of the H-pol probe's electric field to the pump's magnetic field $\theta_{\mathrm{EH}_{\mathrm{prb}} B_{\text {pump }}}$ [Fig. 10(b)] remains $90^{\circ}$ for drift motion along the $x$ axis but does also vary by approximately $\pm 30^{\circ}$ for drift motion in the $z$ direction up to a gamma factor of $\sim 1.25$, but not enough such that the coupling to cyclotron motion is highly suppressed. Most importantly, the angle of the V-pol probe's electric field to the pump's magnetic field $\theta_{\mathrm{EV}_{\mathrm{prb}} B_{\text {pump }}}$ [Fig. 10(c)] remains $0^{\circ}$ for drift motion along the $x$ axis which explains the lack of strong refractive index modulation along the $x$ axis in Figs. 9(b) and 9(d) and reveals the origin of the on-axis break in the V-pol experimental shadowgrams of the RECR half-ring signal seen in Fig. 4(b). For drift motion of the electrons with a strong $z$ component, the V-pol probe's orientation to the pump's magnetic field in the drift frame of the electrons transforms to include an H-pol component ( $x$ component) meaning a coupling to the local cyclotron motion of the electrons. This explains the appearance of large changes in the refractive index of the plasma as seen by the $\mathrm{V}$-pol probe in regions of the plasma where the electrons' drift motion is away from the $x$ axis. The appearance of the on-axis break in the diffraction rings as well as the appearance of the asymmetric signal previously described conveys the importance of a relativistic treatment of the system, especially when dealing with highly anisotropic electron velocity distributions.

\section{CONCLUSION}

In this paper, we have shown evidence and analysis of relativistic electron-cyclotron resonances present in magnetized plasma due to the relativistic drift and cyclotron motion of the plasma electrons in the vicinity of a relativistically intense pump laser using both experimental and simulation data. In the case of LWFA, three signals arising from RECRs can be identified and used to nondestructively track, in situ, the evolution of the linearly polarized laser driver's intensity profile when imaged with a properly selected polarized probe beam. With further development, this technique could constitute the basis of a nondestructive diagnostic for laser or particle beams in plasma-based accelerators. Furthermore, for scenarios comprising relativistic, magnetized plasmas as in fusion research or astrophysics, our method based on RECRs might prove to be an effective diagnostic delivering detailed insight into the underlying physics of such plasmas. 


\section{ACKNOWLEDGMENTS}

B. Beleites and F. Ronneberger contributed by operating the JETI 40 laser facility. M. B. S. and E. S. thank Laurent Gremillet for his valuable scientific support and discussions. The authors gratefully acknowledge the following open-source projects: NumPy [45], MATPLOTLIB [46], and IPYTHON [47]. Simulations were performed using EPOCH code [39] with HPC resources provided by Grand Équipement National De Calcul Intensif (GENCI) A0060507594 and using vsim code [43] with HPC resources provided by the National Energy Research Scientific Computing Center supported by DOE DE-AC0205CH11231. This work was supported by the Bundesministerium für Bildung und Forschung (BMBF) Grants No. 05P15SJFA1, No. 05K16SJC, and No. 05K19SJC as well as the Swedish Research Council, Grant No. 2016-05012.

[1] C. G. R. Geddes, J. Van Tilborg, E. Esarey, C. B. Schroeder, D. Bruhwiler, C. Nieter, J. Cary, and W. P. Leemans, High-quality electron beams from a laser wakefield accelerator using plasma-channel guiding, Nature (London) 431, 538 (2004).

[2] J. Faure, Y. Glinec, A. Pukhov, S. Kiselev, S. Gordienko, E. Lefebvre, J. P. Rousseau, F. Burgy, and V. Malka, A laserplasma accelerator producing monoenergetic electron beams, Nature (London) 431, 541 (2004).

[3] S. P. D. Mangles, C. D. Murphy, Z. Najmudin, A. G. R. Thomas, J. L. Collier, A. E. Dangor, E. J. Divall, P. S. Foster, J. G. Gallacher, C. J. Hooker et al., Monoenergetic beams of relativistic electrons from intense laser-plasma interactions, Nature (London) 431, 535 (2004).

[4] M. Litos, E. Adli, W. An, C. I. Clarke, C. E. Clayton, S. Corde, J. P. Delahaye, R. J. England, A. S. Fisher, J. Frederico et al., High-efficiency acceleration of an electron beam in a plasma wakefield accelerator, Nature (London) 515, 92 (2014).

[5] A. J. Gonsalves, K. Nakamura, J. Daniels, C. Benedetti, C. Pieronek, T. C. H. De Raadt, S. Steinke, J. H. Bin, S. S. Bulanov, J. Van Tilborg et al., Petawatt Laser Guiding and Electron Beam Acceleration to $8 \mathrm{GeV}$ in a Laser-Heated Capillary Discharge Waveguide, Phys. Rev. Lett. 122, 084801 (2019).

[6] A. Aschikhin, C. Behrens, S. Bohlen, J. Dale, N. Delbos, L. Di Lucchio, E. Elsen, J. H. Erbe, M. Felber, B. Foster et al., The FLASHForward facility at DESY, Nucl. Instrum. Methods Phys. Res., Sect. A 806, 175 (2016).

[7] E. Adli, A. Ahuja, O. Apsimon, R. Apsimon, A.-M. Bachmann, D. Barrientos, F. Batsch, J. Bauche, V. K. B. Olsen, M. Bernardini et al., Acceleration of electrons in the plasma wakefield of a proton bunch, Nature (London) 561, 363 (2018).

[8] R. B. Fiorito, A. G. Shkvarunets, T. Watanabe, V. Yakimenko, and D. Snyder, Interference of diffraction and transition radiation and its application as a beam divergence diagnostic, Phys. Rev. ST Accel. Beams 9, 052802 (2006).

[9] J. Van Tilborg, C. Lin, K. Nakamura, A. J. Gonsalves, N. H. Matlis, T. Sokollik, S. Shiraishi, J. Osterhoff, C. Benedetti, C. B. Schroeder et al., Long-range persistence of femtosecond modulations on laser-plasma-accelerated electron beams, AIP Conf. Proc. 1507, 774 (2012).

[10] S. Wesch, B. Schmidt, C. Behrens, H. Delsim-Hashemi, and $\mathrm{P}$. Schmüser, A multi-channel $\mathrm{THz}$ and infrared spectrometer for femtosecond electron bunch diagnostics by single-shot spectroscopy of coherent radiation, Nucl. Instrum. Methods Phys. Res., Sect. A 665, 40 (2011).

[11] A. Buck, K. Zeil, A. Popp, K. Schmid, A. Jochmann, S. D. Kraft, B. Hidding, T. Kudyakov, C. M. S. Sears, L. Veisz et al., Absolute charge calibration of scintillating screens for relativistic electron detection, Rev. Sci. Instrum. 81, 033301 (2010).

[12] Y. Glinec, J. Faure, A. Guemnie-Tafo, V. Malka, H. Monard, J. P. Larbre, V. De Waele, J. L. Marignier, and M. Mostafavi, Absolute calibration for a broad range single shot electron spectrometer, Rev. Sci. Instrum. 77, 103301 (2006).

[13] M. Kaluza, H.-P. Schlenvoigt, S. Mangles, A. Thomas, A. Dangor, H. Schwoerer, W. Mori, Z. Najmudin, and K. Krushelnick, Measurement of Magnetic-Field Structures in a Laser-Wakefield Accelerator, Phys. Rev. Lett. 105, 115002 (2010).

[14] B. Walton, A. E. Dangor, S. P. D. Mangles, Z. Najmudin, K. Krushelnick, A. G. R. Thomas, S. Fritzler, and V. Malka, Measurements of magnetic field generation at ionization fronts from laser wakefield acceleration experiments, New J. Phys. 15, 025034 (2013).

[15] A. Buck, M. Nicolai, K. Schmid, C. M. S. Sears, A. Sävert, J. M. Mikhailova, F. Krausz, M. C. Kaluza, and L. Veisz, Real-time observation of laser-driven electron acceleration, Nat. Phys. 7, 543 (2011).

[16] P. Dong, S. A. Reed, S. A. Yi, S. Kalmykov, Z. Y. Li, G. Shvets, N. H. Matlis, C. McGuffey, S. S. Bulanov, V. Chvykov, G. Kalintchenko et al., Holographic visualization of laser wakefields, New J. Phys. 12, 045016 (2010).

[17] A. Sävert, S. P. D. Mangles, M. Schnell, E. Siminos, J. M. Cole, M. Leier, M. Reuter, M. B. Schwab, M. Möller, K. Poder et al., Direct Observation of the Injection Dynamics of a Laser Wakefield Accelerator Using Few-Femtosecond Shadowgraphy, Phys. Rev. Lett. 115, 055002 (2015).

[18] C. J. Zhang, J. F. Hua, X. L. Xu, F. Li, C.-H. Pai, Y. Wan, Y. P. Wu, Y. Q. Gu, W. B. Mori, C. Joshi, and W. Lu, Capturing relativistic wakefield structures in plasmas using ultrashort high-energy electrons as a probe, Sci. Rep. 6, 29485 (2016).

[19] C. J. Zhang, J. F. Hua, Y. Wan, C. H. Pai, B. Guo, J. Zhang, Y. Ma, F. Li, Y. P. Wu, H. H. Chu et al., Femtosecond Probing of Plasma Wakefields and Observation of the Plasma Wake Reversal Using a Relativistic Electron Bunch, Phys. Rev. Lett. 119, 064801 (2017).

[20] G. Pariente, V. Gallet, A. Borot, O. Gobert, and F. Quéré, Space-time characterization of ultra-intense femtosecond laser beams, Nat. Photonics 10, 547 (2016).

[21] M. Röhrs, C. Gerth, H. Schlarb, B. Schmidt, and P. Schmüser, Time-resolved electron beam phase space 
tomography at a soft x-ray free-electron laser, Phys. Rev. ST Accel. Beams 12, 050704 (2009).

[22] T. Matsuoka, C. McGuffey, P. G. Cummings, Y. Horovitz, F. Dollar, V. Chvykov, G. Kalintchenko, P. Rousseau, V. Yanovsky, S. S. Bulanov et al., Stimulated Raman Side Scattering in Laser Wakefield Acceleration, Phys. Rev. Lett. 105, 034801 (2010).

[23] A. G. R. Thomas, S. P. D. Mangles, Z. Najmudin, M. C. Kaluza, C. D. Murphy, and K. Krushelnick, Measurements of Wave-Breaking Radiation from a Laser-Wakefield Accelerator, Phys. Rev. Lett. 98, 054802 (2007).

[24] M. Bornatici, R. Cano, O. De Barbieri, and F. Engelmann, Electron cyclotron emission and absorption in fusion plasmas, Nucl. Fusion 23, 1153 (1983).

[25] I. B. Bernstein and D. C. Baxter, Relativistic theory of electron cyclotron resonance heating, Phys. Fluids 24, 108 (1981).

[26] H. Park, E. Mazzucato, T. Munsat, C. W. Domier, M. Johnson, N. C. Luhmann, J. J. Wang, Z. Xia, I. G. J. Classen, A. J. H. Donne, and M. J. van de Pol, Simultaneous microwave imaging system for density and temperature fluctuation measurements on TEXTOR, Rev. Sci. Instrum. 75, 3787 (2004).

[27] S. Kuschel, M. B. Schwab, M. Yeung, D. Hollatz, A. Seidel, W. Ziegler, A. Sävert, M. C. Kaluza, and M. Zepf, Controlling the Self-Injection Threshold in Laser Wakefield Accelerators, Phys. Rev. Lett. 121, 154801 (2018).

[28] M. B. Schwab, A. Sävert, O. Jäckel, J. Polz, M. Schnell, T. Rinck, L. Veisz, M. Moeller, P. Hansinger, G. G. Paulus, and M.C. Kaluza, Few-cycle optical probe-pulse for investigation of relativistic laser-plasma interactions, Appl. Phys. Lett. 103, 191118 (2013).

[29] D. Adolph, M. Moeller, J. Bierbach, M. Schwab, A. Saevert, M. Yeung, A. M. Sayler, M. Zepf, M. C. Kaluza, and G. G. Paulus, Real-time, single-shot, carrier-envelopephase measurement of a multi-terawatt laser, Appl. Phys. Lett. 110, 081105 (2017).

[30] J. A. Bittencourt, Fundamentals of Plasma Physics, 3rd ed. (Springer-Verlag, Berlin, 2004).

[31] D. A. Gurnett and A. Bhattacharjee, Introduction to Plasma Physics: With Space, Laboratory and Astrophysical Applications, 2nd ed. (Cambridge University Press, Cambridge, England, 2017).

[32] E. S. Sarachik and G. T. Schappert, Classical theory of the scattering of intense laser radiation by free electrons, Phys. Rev. D 1, 2738 (1970).
[33] H. Unz, Relativistic magneto-ionic theory for drifting plasma in longitudinal direction, Phys. Rev. 146, 92 (1966).

[34] U.E. Schroeder, Spezielle Relativitaetstheorie (Verlag Europa-Lehrmittel, Vollmer $\mathrm{GmbH} \& \mathrm{Co}$. KG, HaanGruiten, 1994).

[35] F. V. Hartemann, High-Field Electrodynamics (CRC Press LLC, Boca Raton, Florida, 2002).

[36] M. Bornatici, Theory of electron cyclotron absorption of magnetized plasmas, Plasma Phys. 24, 629 (1982).

[37] V. Erckmann and U. Gasparino, Electron cyclotron resonance heating and current drive in toroidal fusion plasmas, Plasma Phys. Controlled Fusion 36, 1869 (1994).

[38] C. Thaury, F. Quéré, J. P. Geindre, A. Levy, T. Ceccotti, P. Monot, M. Bougeard, F. Réau, P. D’Oliveira, P. Audebert, R. Marjoribanks, and P. Martin, Plasma mirrors for ultrahigh-intensity optics, Nat. Phys. 3, 424 (2007).

[39] T. D. Arber, K. Bennett, C. S. Brady, A. LawrenceDouglas, M. G. Ramsay, N. J. Sircombe, P. Gillies, R. G. Evans, H. Schmitz, A. R. Bell, and C. P. Ridgers, Contemporary particle-in-cell approach to laser-plasma modelling, Plasma Phys. Controlled Fusion 57, 113001 (2015).

[40] E. Siminos, S. Skupin, A. Sävert, J. M. Cole, S. P. D. Mangles, and M. C. Kaluza, Modeling ultrafast shadowgraphy in laser-plasma interaction experiments, Plasma Phys. Controlled Fusion 58, 065004 (2016).

[41] E. Esarey, C. B. Schroeder, B. A. Shadwick, J. S. Wurtele, and W. P. Leemans, Nonlinear Theory of Nonparaxial Laser Pulse Propagation in Plasma Channels, Phys. Rev. Lett. 84, 3081 (2000).

[42] C. D. Decker, W. B. Mori, K.-C. Tzeng, and T. Katsouleas, The evolution of ultra-intense, short-pulse lasers in underdense plasmas, Phys. Plasmas 3, 2047 (1996).

[43] C. Nieter and J. R. Cary, VORPAL: a versatile plasma simulation code, J. Comput. Phys. 196, 448 (2004).

[44] S. V. Bulanov, F. Pegoraro, A. M. Pukhov, and A. S. Sakharov, Transverse-Wake Wave Breaking, Phys. Rev. Lett. 78, 4205 (1997).

[45] S. Van Der Walt, S. C. Colbert, and G. Varoquaux, The NumPy array: A structure for efficient numerical computation, Comput. Sci. Eng. 13, 22 (2011).

[46] J. D. Hunter, Matplotlib: A 2D Graphics Environment, Comput. Sci. Eng. 9, 90 (2007).

[47] F. Pérez and B.E. Granger, IPython: A system for interactive scientific computing, IEEE J. Mag. 9, 21 (2007). 